\title{
Evaluation of AVO attributes derived from Angle sub stacks
}

\author{
Roy TADA $^{1}$ and Yasutaka ICHIE ${ }^{1}$ \\ ${ }^{1}$ Teikoku Oil Co., Ltd.
}

\begin{abstract}
Amplitude-versus-offset (AVO) attribute analysis becomes a defact-standard technique for the contemporary hydrocarbon exploration. A basic product for this analysis is angle sub stacks, such as so-called near and far, and sometime middle in order to evaluate an amplitude change along offset or incident angle. Detail study of AVO interpretation is facilitated by crossplotting AVO intercept (P0) and gradient (G) under Shuey's two term approximation. Further elastic attributes are derived basically from those P0 and G.

Estimation of the $\mathrm{P} 0$ and $\mathrm{G}$ is normally derived via least-square fit of a prestack-migrated seismic gather, which is transformed from offset into incident angle. A partial stack technique is commonly introduced to enhance signal-to-noise ratio $(\mathrm{S} / \mathrm{N})$ anticipating better estimation. This ultimate case of the partial stack is the angle sub stack utilizing for amplitude study at an interpretation.

In this paper, we are using synthetic and real seismic data contaminated by Gaussian noise to demonstrate that P0 and $\mathrm{G}$ estimation based on angle sub stacks is as reliable as computed on a full gather. This implies our standard interpretation data in our hands will be enough for in-house calculation of P0 and G, which can be done by an interpretation workstation, and this contributes budget saving for further $\mathrm{P} 0$ and $\mathrm{G}$ based processing.
\end{abstract}

\section{INTRODUCTION}

Amplitude-versus-offset (AVO) method was developed to help delineate the fluid content of reservoirs (Ostrander, 1984). AVO analysis has now become a routine for interpreting the variation of reflection amplitudes with angle of incidence in terms of changes in lithology and/or fluid contents. Shuey (1985) showed that for angles of incidence $\theta$ less than about $25^{\circ} \sim 35^{\circ}$, the $\mathrm{P}$-wave reflection amplitude from a planar interface between two elastic media can be approximated by

$$
P(\theta)=P 0+G \sin ^{2} \theta .
$$

Accurate AVO analysis relies on good quality of seismic data. Therefore, most of the current AVO inversion software has utility of angle sub stack as AVO pre-conditioning. Generally, using more angle sub stacks gives a more detailed description of AVO, but at the same time the signal-to-noise ratio $(\mathrm{S} / \mathrm{N})$ of individual sub stacks decreases.

Yin and Pillet (2006) studied how many angle sub stacks should be used to get the best inversion result and concluded 4 angle sub stacks as the optimal number for both acoustic and shear impedance, while 2 sub stacks gave a best result at the acoustic impedance. Rauch-Davis and
Portniaguine (2004) discussed elastic attribute inversion using 3 angle sub stacks.

Those papers indicate 3 angle sub stacks are good enough for further inversion. It is important to remember that for a typical seismic project, alongside with full fold stack an interpreter is provided with 3 angle sub stacks and herewith - an opportunity to estimate AVO attributes on an interpretation workstation. This study demonstrates how the 3 angle sub stacks provide comparative AVO attributes to prestack AVO processing.

\section{SYNTHETIC DATA}

The data set are 1) Gaussian distribution along offset $\left(\operatorname{Sin}^{2} \theta\right)$, 2) Zoeppritz approximation of well log data at gas bearing sand and shale sequence 3 ) full wave finite difference simulation of well data. Those well data are presented by P-wave, S-wave and density logs. P0 and G are calculated by full angle gather ranged from 5 to 35 degrees with 2 angle sub stacks (5-20 and 20-35 degrees) and 3 angle sub stacks (5-15, 15-25 and 25-35 degrees), which are considered as standard products of angle sub stacks associated with stack data at an interpretation. 


\section{(1) Gaussian distribution}

Figure 1 shows a Gaussian distribution of amplitude along offset $\left(\operatorname{Sin}^{2} \theta\right)$, which is marked red dots. Two angle sub stacks are of 2 stacks (green cross) which are simple amplitude average along angles from 5 to 20 and from 20 to 35 and of 3 stacks (blue star) of 5-15, 15-25 and 25-30 degrees.

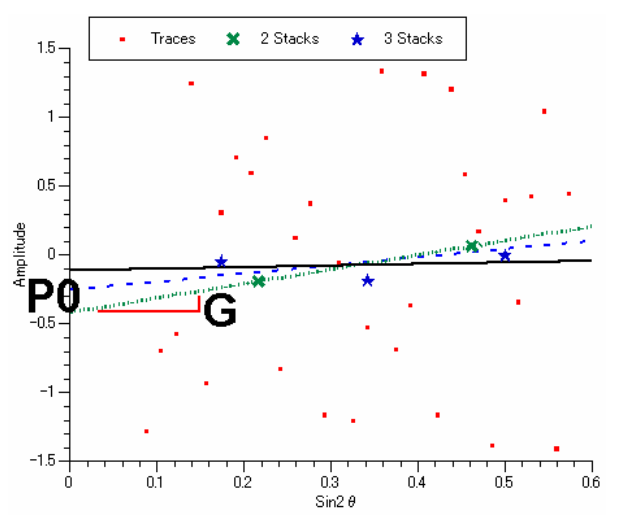

Figure 1 Gaussian distribution of amplitude along offset (black dot) and angle sub stacks of those amplitude average (green cross and blue star).

Linear regression of those 3 cases provides different $P 0$ and $G$ values, while $P 0=G=0$ is expecting. These differences bring tight hesitation to utilize any stacks for a precise calculation. Is it true that the sub stacked data give such difference?

\section{(2) Statistical evaluation of Gaussian simulations}

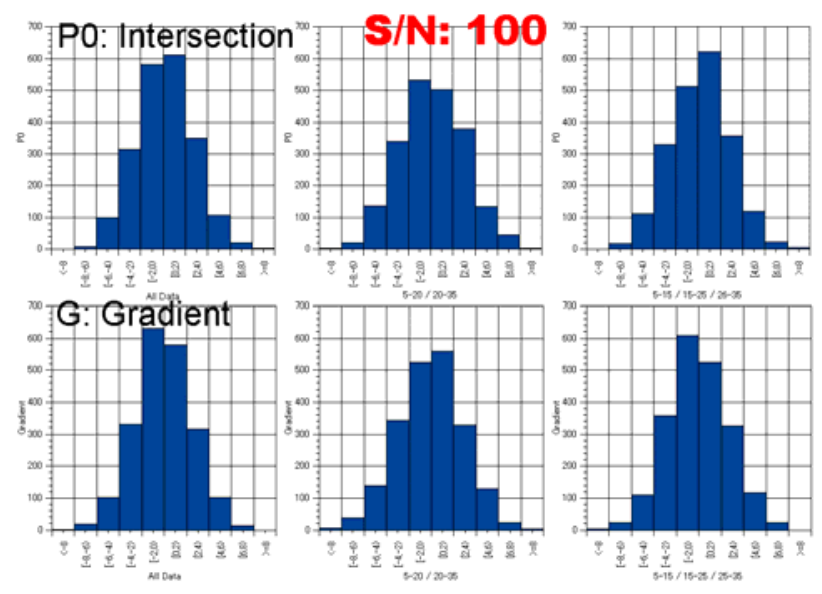

Figure 2 P0 and G distribution for gather data (left), 2 sub stacks (mid) and 3 sub stacks (right) for $\mathrm{S} / \mathrm{N}=100$ case.

We calculate 2048 times of the Gaussian simulations and make 2 and 3 angle sub stacks. Figure 2 shows the distribution of P0 and G results for gather data, 2 angle sub stacks and 3 angle sub stacks. This $\mathrm{S} / \mathrm{N}=100$ is defined \pm 0.01 amplitude deviation from zero line.

It is clear that those three types of calculations give poor segregation on the $\mathrm{P} 0$ or $\mathrm{G}$ distributions in such a low noise condition. For more clarification, we compute another 2048 times, but $\mathrm{S} / \mathrm{N}$ is as very low as 4 , which has amplitude variation about \pm 0.25 (see Figure 3).

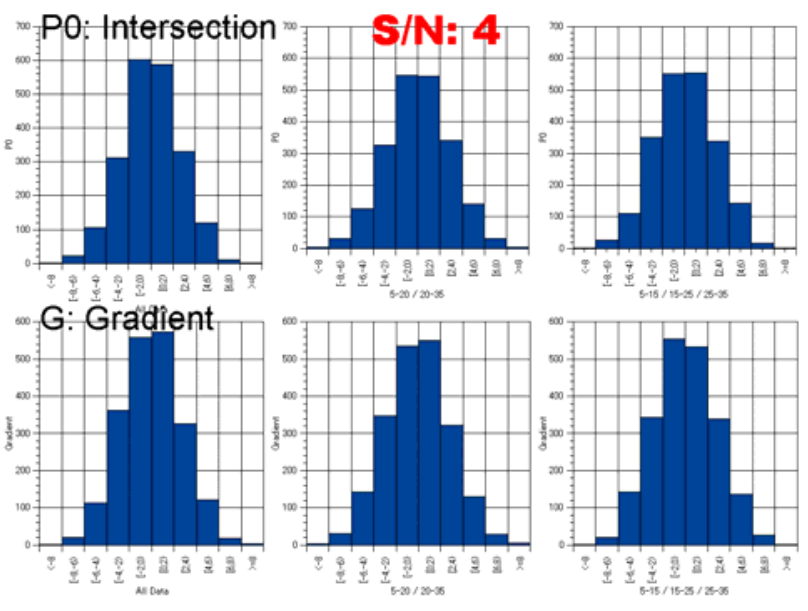

Figure $3 \mathrm{P} 0$ and $\mathrm{G}$ distribution for $\mathrm{S} / \mathrm{N}=4$ case.

Although there is hardly identified a distribution from any other distributions in both Figure $\mathbf{2}$ and 3, it is only aware of 2 angle sub stacks of $\mathrm{S} / \mathrm{N}=100$ case. Usage of full gather is now realized to be not the best selection for the P0 and G estimation. Deviation from zero value, which is expected, is due to outliers' effects generated by noise. While it is expected that the stacking shall be worked to eliminate the effect, how come the stacking effect such as narrowing the distribution shape is not reflected on to the 2 or 3 angle sub stack from Figures 2 and 3 ?

\section{(3) Well log synthetic seismogram a) Zoeppritz simulation}

A well synthetic seismic simulation is generated using sonic, shear wave sonic and density logs. First set is simulated angle gather from 5 to 50 degrees using Zoeppritz approximation. Figure 4 is the result of the simulation and its AVO attributes in terms of full gather, 2 angle sub stacks and 3 angle sub stacks within the range of 5 to 35 degrees, which trace is marked in thick line on the left panel of angle gather.

Shallow strong reflection indicates AVO response in Figure $\mathbf{4}$ and is supported by well information as gas bearing. Lower strong event is a brine sand reflection and no AVO response is simulated as expected.

This AVO attribute of P0 and G results found in 
right two panels of the figure are most identical and reinforces the confirmation of the result of the Gaussian simulation. It may be concerned to be noise free case. Amount of noise will cause the differences on the attribute and effect of stack shall be aware when noise is added.

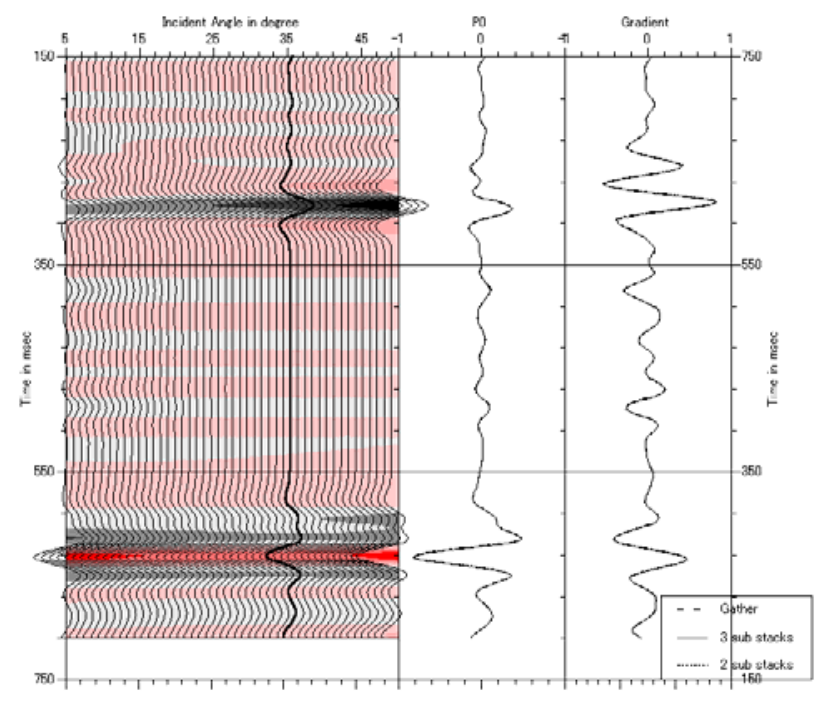

Figure 4 Noise free case of well log synthetic seismogram of Zoeppritz approximation. Thick trace in the left panel indicates 35 degree angle trace, which is upper limit of P0 and G calculation. Dashed line in right two panels indicates calculation of full angle traces ranging 5 to 35 degree, solid line is result of 3 angle sub stack of $5-15,15-25$ and 25-35, and dotted line is 2 angle sub stacks of 5-20 and 20-35. Rest of the figures is consistent to this notation. Sample rate is $1 \mathrm{~ms}$.

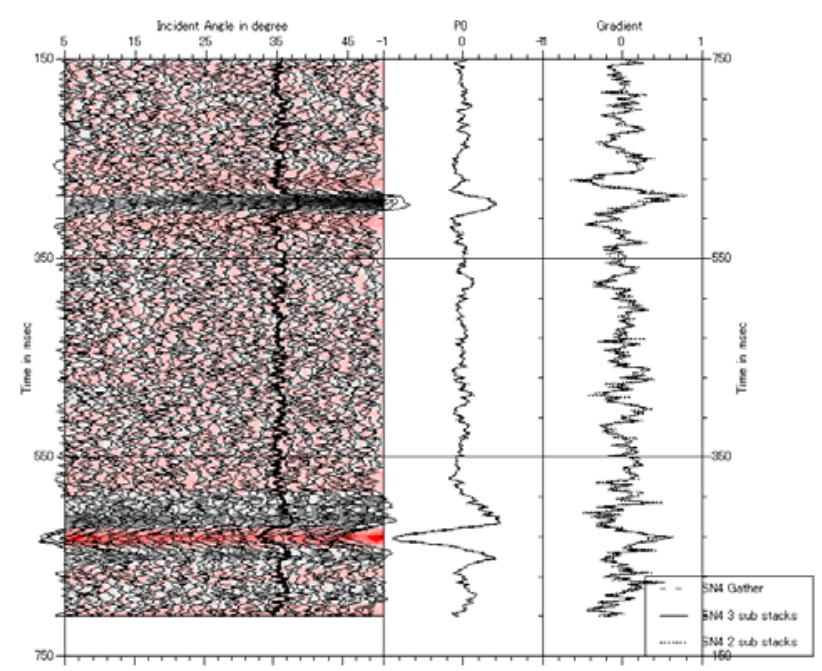

Figure 5 Noise added on Figure 4. $\mathrm{S} / \mathrm{N}$ is 4 . Sample rate is $1 \mathrm{~ms}$.

Figure 5 is the case of $\mathrm{S} / \mathrm{N}=4$, which is $25 \%$ noise added. AVO attributes are as noisy as angle gather, but they follow closely each other. It is remarked that $\mathrm{P} 0$ is less sensitive under a noise condition than $\mathrm{G}$. is.

\section{b) Full wave finite difference simulation}

It is hard to find difference due to stack effect on the synthetic seismogram. This is supposed that the result is to be utilized an ideal approximation and the process of noise adding is as same as the previous Gaussian simulation does.

We change the simulation to a full wave finite difference as shown in Figure 6, which is already noise added being $\mathrm{S} / \mathrm{N}=4$, where we have a weak AVO at 2410ms. The input well log data is not same as the study on the Zoeppritz simulation. The transformation from offset simulation to angle is done by Walden's equation (Walden, 1991), after smoothing of interval velocity, which is sonic log.

The result is as noisy as the case of Zoeppritz and it is confirmed that P0 of intersection has less deviation in each other than $G$ of gradient shows.

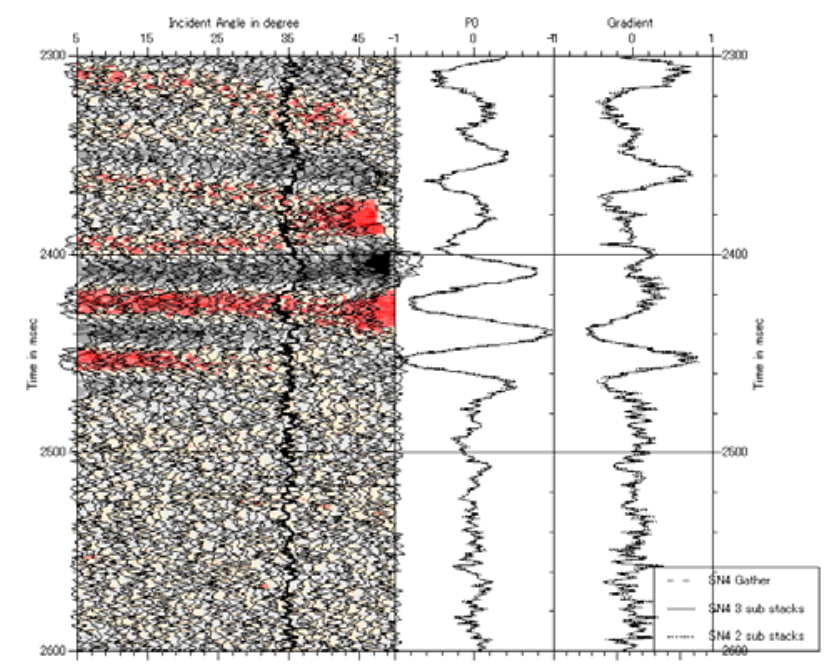

Figure 6 Full wave finite difference simulations of other well log data. Sample rate is $1 \mathrm{~ms}$.

\section{SEISMIC DATA}

The data examined in these two real examples are chosen to illustrate important points regarding estimation of AVO attributes.

\section{(1) Shallow AVO data}

The first example is a shallow AVO case and original $\mathrm{S} / \mathrm{N}$ is very high. Figure $\mathbf{7}$ is the result of $25 \%$ noise addition, which is assumed to be $\mathrm{S} / \mathrm{N}=4$, on to the very clean shallow data set. There is a positive and negative reflection pair of AVO at around $1100 \mathrm{~ms}$ in the left panel of angle gather. The thick trace indicates an angle limitation of AVO attribute calculation as shown in previous figures.

Focusing on the AVO attribute deviation, all 
angle and 3 angle sub stacks are identical, but not 2 angle sub stacks. While this observation of 2 angle sub stacks becomes conscious, magnitude of the deviation itself is not sizable as being recognized. We can rather say that all of the attribute results come close to each other and any of the attribute can be properly represented the AVO.

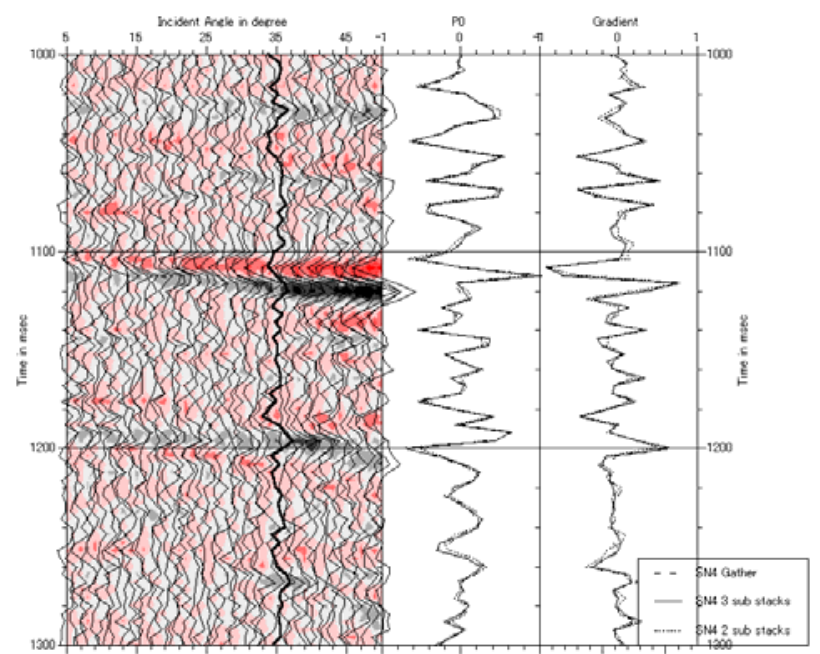

Figure 7 Shallow AVO case adding 25\% noise to be $\mathrm{S} / \mathrm{N}=4$. Sample rate is $4 \mathrm{~ms}$.

\section{(2) Deeper AVO data}

Last example is deeper than the Figure 7 in AVO occurrence and more noisy data (see Figure 8). We can see the AVO pair just below 1,600 ms. Those AVO indicate weak amplitude of P0, therefore P0 deviation will be anticipated. In addition, smoothness of AVO attributes on Figure 8, comparing with Figure 7 at same window and same length, is supposed to be the difference of processing sample rate.

This result reconfirms the previous observation of 1) all attributes mimic each other and 2) less deviation on P0 calculation than gradient shows. This behavior of $\mathrm{P} 0$ gives an advantage to utilize angle sub stacks for presence of weak amplitude P0, which is classified as Class II AVO (Rutherford and Williams, 1989; Castagna and Swan, 1997).

\section{DISCUSSION}

Due to the size of each figure on the paper, it is hard to make detail study such faint differences. In order to magnify the differences, we will discuss if these differences are meaningful for the AVO attribute computation and further inversion based on the attributes using a crossplot.

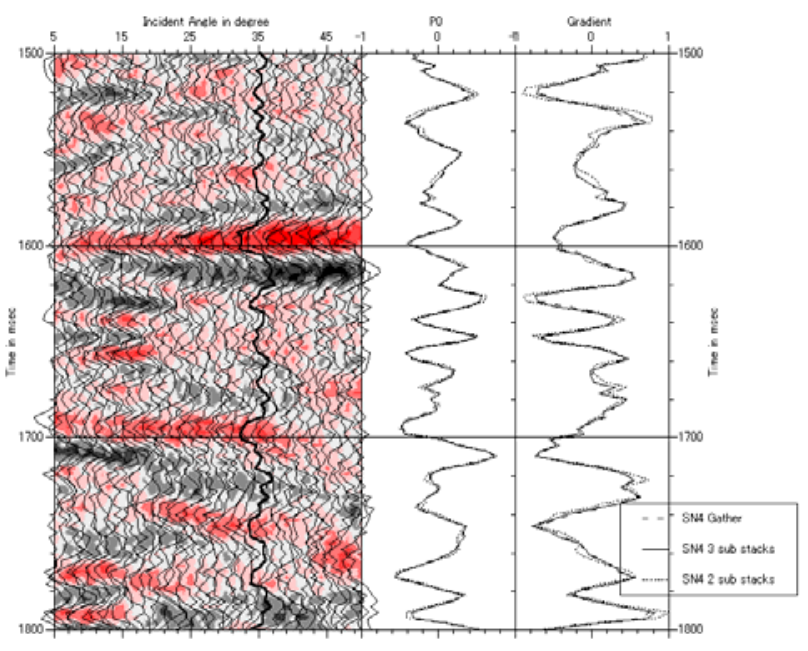

Figure 8 Deeper AVO case adding 25\% noise $(\mathrm{S} / \mathrm{N}=4)$. Sample rate is $3 \mathrm{~ms}$.

\section{(1) Evaluation of crossplot}

Figure 9 shows crossplots of gradients calculations for the interval between 1500 and 1800 of full gather, 2 angle sub stacks and 3 angle sub stacks at $\mathrm{S} / \mathrm{N}=4$ case versus full gather of noise free case, which is regarded as those ideal result. It is clear that both of the seismic data of deeper AVO case and the full wave finite difference simulation of well log show scatters or outliers created by addition of noise. Linear regression of each gradient trend is, however, close each other, especially the full wave finite difference simulation.
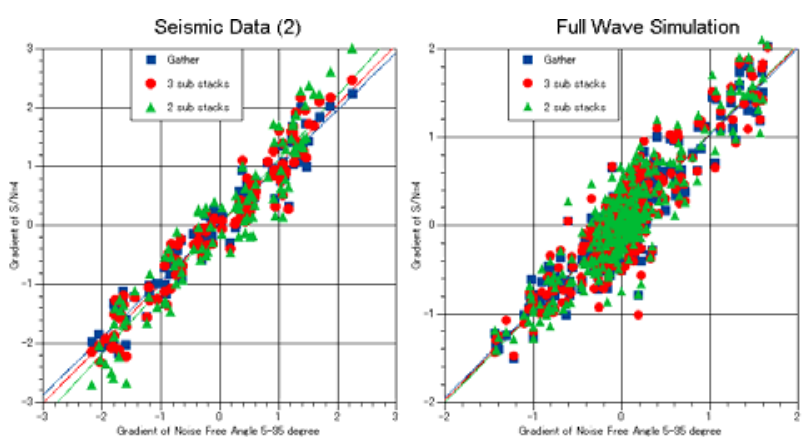

Figure 9 Crossplots of gradient calculations for three different data of $\mathrm{S} / \mathrm{N}=4$ angle sub stacks versus noise-free full angle gather, which shall represent the ideal result.

Before go further into the conclusion, we make another clarification for the consistency of AVO attribute calculation under stack process.

\section{(2) Amplitude study along angle gahter}

Figure $\mathbf{1 0}$ is amplitude study along angle or offset at a pair of AVO found at around $1610 \mathrm{~ms}$. on Figure 8. Black dots on the crossplot are represented the original seismic data shown on the 
top right. Blue filled triangles are of noise addition $(\mathrm{S} / \mathrm{N}=4)$ shown on the bottom right seismic. Green filled circles are of locations from 3 angle sub stacks, which are summed traces in ranges of 5-15, 15-25 and 25-35 degrees, and red crosses are from 2 angle sub stacks, which are averages at 5-20 and 30-35 degrees.

At both AVO cases of trough and peak traces, positions of both 2 and 3 angle sub stacks are no much deviated from the linear regressions of full angle gather of original data, which are shown with black lines on the crossplot, while the noisy angle gather data shows wide scatterings or outliers (blue filled triangles). Another remark is conscious that all of the linear regressions, which color is correspond to those makers, are close together even if the noisy full angle gather (blue filled circles and lines). This gives consistent results of gradient calculations on the left panel of Figure 8.

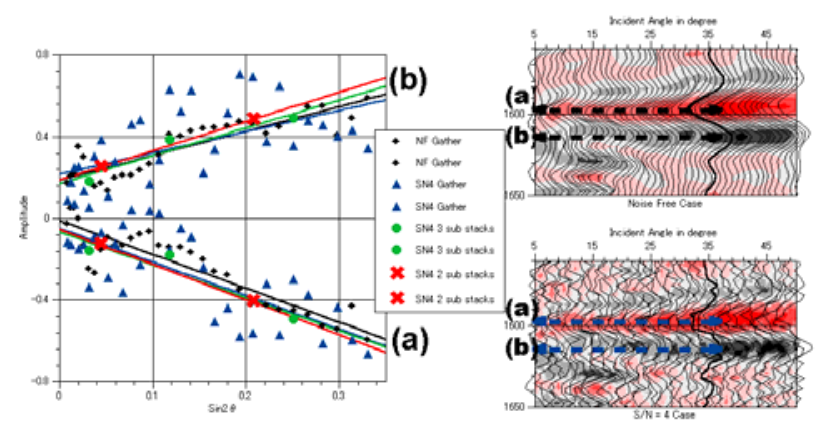

Figure 10 Crossplot of angle versus amplitude at a pair of AVO traces and those angle gathers for both original and addition of noise being $\mathrm{S} / \mathrm{N}=4$.

The evaluation of the crossplots indicates the gradient calculation is very stable even at very noisy data such as $\mathrm{S} / \mathrm{N}=4$. It is remarkable that P0 calculation is more consistent for all simulations and seismic data than the gradient is. So that the angle stack data can be appointed to alternative data for AVO attribute calculation.

\section{CONCLUSION}

Those observations suggest the following strategy toward AVO attribute processing of stacked data:

(i) Different numbers of angle sub stacks (2 and 3 angle sub stacks) are generated from the angle range 5 to 35 degrees for AVO attribute calculations. Those attributes are compared with angle gather, to which is not applied any stack process.

(ii) Attributes from the 2 or 3 angle sub stacks give quite competitive result to the calculation from the full angle gather. (iii) This observation is consistent toward all of the well log synthetics and seismic data.

(iv) Noisy traces of $\mathrm{S} / \mathrm{N}=4$ give rise to no confusion at the attributes calculation from clean case. This may imply the stacking effect, which is usually resulted better $\mathrm{S} / \mathrm{N}$ than before, is not observed on the AVO attribute process.

(v) Because of the linear regression process on AVO attribute calculation, summation is involved in the regression, so that the effect of stack is done in the regression and the result shall be statistically very much similar to each other (Figure 2 and $\mathbf{3}$ ).

(vi) The number of angle sub stacks is similar for 2 and 3 cases, but we practically recommend the 3 angle sub stacks for AVO attribute calculation because of better match to the result of the full angle gather. However, we must recognize that the optimum number of angle sub stacks is data-set dependent.

(vii) This number of angle sub stacks is standard products when we start interpretation. Therefore, this implies that we can calculate AVO attribute within our interpretation workstation with very comparative resolution to the full angle gather computation, which is very heavy process because of domain in pre-stack.

(viii) Main disturbance of calculation result is considered to be miss alignment of AVO events and is not noise. The alignment problem due to NMO velocity is hardly reviewed after the angle stack process. However, the issue lies on the gather itself and it will not be solved even by use of the full gather for AVO attributes. Zero-phase application before AVO analysis is recommended considering this alignment and change of wavelet along offset or angle.

ACKNOWLEDGMENT: The authors thank the President and Board of Director of Teikoku Oil Co., Ltd, especially Mr. Masaharu Sano, for permission to publish the paper. The insight comments and many fruitful discussions with Mr. Swee Leng Ng of WesternGeco and Mr. Anatoly Osadchuk of Direct Seismic Processing Services Pty Ltd both in Perth, Australia are gratefully acknowledged.

\section{REFERENCES}

1) Ostrander, W. J., 1984., Plane-wave reflection coefficients for gas sands at non-normal angles of incidence, Geophysics, 49, 1637- 1648.

2) Shuey, R. T., 1985., A simplification of Zoeppritz equation, Geophysics, 50, 609- 614.

3) Yin, Y., and Pillet, W., 2006., Seismic data preparation for improved elastic inversion of 
angle sub stacks, 76th Ann International Meeting, Soc. Expl. Geophys., Expanded Abstract, 20422046.

4) Rauch-Davis, M., and Portniaguine, O., 2004., Elastic Attribute Generation From 3 Points Elastic Inversion, OTC 16731.

5) Walden, A. T., 1991., Making AVO sections more robust, Geophysical Prospecting, 39, 915- 942.

6) Rutherford, S. R., and Williams, R. H., 1989., Amplitude-versus-offset variations in gas sands, Geophysics, 54, 680- 688.

7) Castagna, J. P., and Swan, H. W., 1997., Principles of AVO crossplotting, The Leading Edge, 16, 337 - 344. 